\title{
Unusual Peritoneal Carcinomatoses Detected on The Follow Up F-18 FDG PET/CT in the Patient with Breast Cancer
}

\author{
Seok-Nam Yoon* \\ Department of Nuclear Medicine, Dankook University College of Medicine, Republic of Korea
}

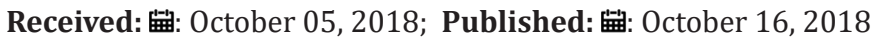

*Corresponding author: Seok-Nam Yoon, Department of Nuclear Medicine, Cheil General Hospital, Women Cancer Center, Dankook University College of Medicine 1-19 mukjung-dong, Jung-gu, Seoul 100-380 Republic of Korea

\begin{abstract}
A 30-year-old woman four years ago underwent left mastectomy due to stage I invasive breast carcinoma with 8mm sized invasive mass, histologic grade $2 / 3$, nuclear grade $2 / 3$ and lymphovascular invasion. She had radiation therapy only without any chemotherapy or hormone therapy. F-18 FDG PET/CT scan underwent for evaluation of recurrent lesion. PET/CT showed multiple peritoneal carcinomatoses in the right / lateral abdomen/right pelvic cavity and in the perihepatic space, large hepatic metastasis in the right lobe of the liver, osteolytic bone metastasis in the right iliac bone, multiple node metastases in the left paraaortic node, left common iliac node and both internal iliac nodes. Large amount of ascites is noted in pelvis and lower abdominal cavity on the abdominal -pelvic CT. Ascite cytology was performed to rule out another malignant lesion due to unusual metastatic findings. Cytology showed positive for malignant cells consistent with metastatic carcinoma from previous breast carcinoma. Immunohistochemical staings with breast cancer and ascites were entirely equivalent.
\end{abstract}

Keywords: Breast Carcinoma; Peritoneal Carcinomatoses; FDG

\section{Introduction}

Generally typical breast cancer metastatization sites are, in order of frequency, bones, liver, lungs and brain, but very rare secondary localizations are the peritoneal cavity. Some study revealed very low incidence of $0.7 \%$. In fact, although peritoneal carcinomatosis usually affects patients with solid intra-abdominal cancers, including those originated by the gastrointestinal tract and those originating by the female gynecologic malignant lesion, it may also derive from breast cancer.

\section{Case Report}

A 30-year-old woman four years ago underwent left mastectomy due to stage I invasive breast carcinoma with $8 \mathrm{~mm}$ sized invasive mass, histologic grade $2 / 3$, nuclear grade $2 / 3$ and lymphovascular invasion. She had radiation therapy only without any chemotherapy or hormone therapy. A-30-years-female patient with breast carcinoma underwent F-18 FDG whole body PET-CT study for evaluation of metastatic lesion. PET/CT (Figures 1-4) showed multiple peritoneal carcinomatoses in the right / lateral abdomen/right pelvic cavity and in the perihepatic space, large hepatic metastasis in the right lobe of the liver, osteolytic bone metastasis in the right iliac bone, multiple node metastases in the left paraaortic node, left common iliac node and both internal iliac nodes. Large amount of ascites is noted in pelvis and lower abdominal cavity on the abdominal -pelvic CT. Ascite cytology was performed to rule out another malignant lesion due to unusual metastatic findings. Aaspiration cytology using ascites revealed positive for malignant cells consistent with metastatic carcinoma. Immunohistochemistry studies including ER, PR, P53, C-Erb-2, EGFR and CK5/6 were entirely. Equivalent cytology showed positive for malignant cells consistent with metastatic carcinoma from previous breast carcinoma.

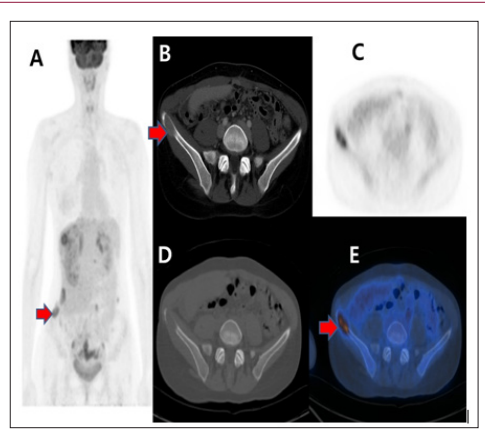

Figure 1: Abdominal pelvic CT with contrast enhancement showed osteolytic lesion in the right iliac bone (B). The maximum intensity projection PET images(A), corresponding transverse PET-CT(CD), fusion(E) showed intense FDG uptake suggesting osteolytic iliac bone metastasis. 


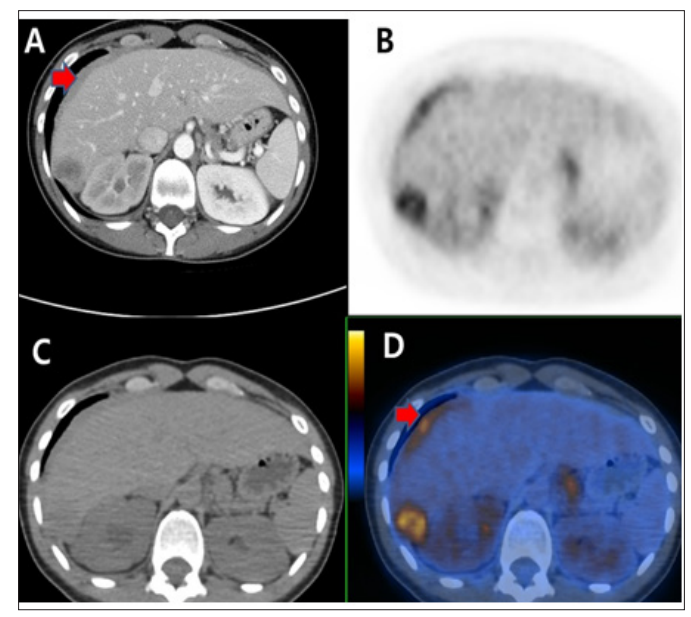

Figure 2: Abdominal pelvic CT with contrast enhancement(A) showed $3.2 \times 2.0 \mathrm{~cm}$ sized central low peripheral enhancing hepatic mass in Rt. liver tip portion and soft tissue lesion with enhancement in the perihepatic space. Liner FDG uptakes along the perihepatic space and focal FDG uptake in the right livers suggesting peritoneal carcinomatoses and hepatic metastasis demonstrated on the F-18 FDG PET-CT (B, C, D).

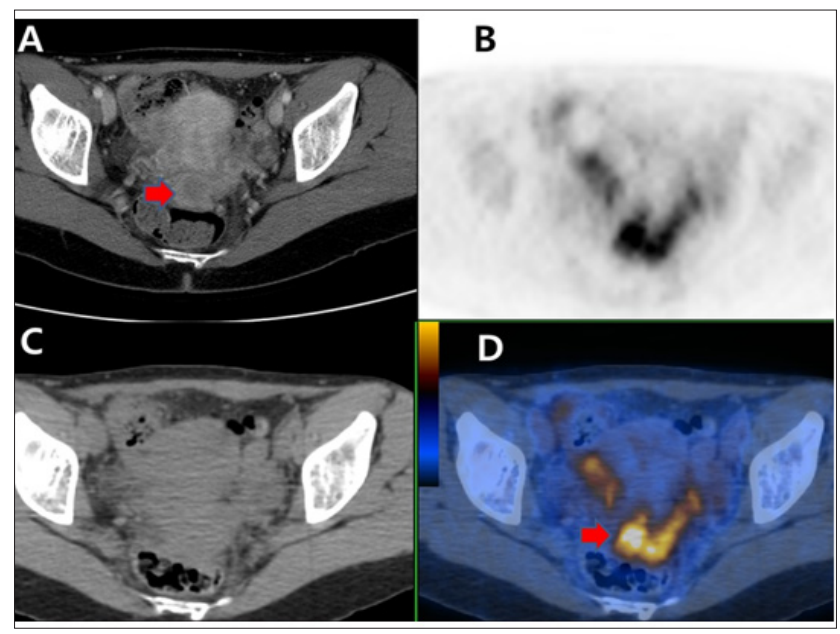

Figure 3: Abdominal pelvic CT with contrast enhancement(A) showed amorphous enhancing soft tissue portion in pelvic cavity. And any definite abnormal finding in the both ovary could not find on the CT. F-18 FDG PET-CT (B, C, D) showed intense FDG uptake in the pelvic lesions with amorphous enhancing soft tissue on the CT. And this could be interpreted as metastatic lesions in the pelvis.

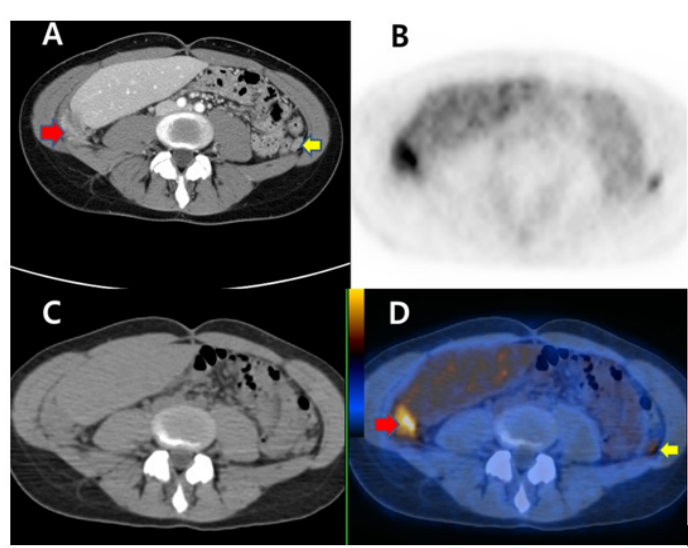

Figure 4: Abdominal pelvic CT with contrast enhancement(A) showed enhancing small peritoneal nodule in left abdominal cavity and large peritoneal nodule in the right abdominal cavity below right liver. Intense focal FDG uptakes in both abdomen suggesting peritoneal carcinomatoses demonstrated on the F-18 FDG PET-CT (BCD). 


\section{Discussion}

Usually peritoneal carcinomatoses may be due to ovarian carcinoma. Peritoneal metastases from breast carcinoma is a rare condition [1-4] Peritoneal carcinomatosis represents a very high mortality rate. F-18 FDG PET/CT show very useful tool for evaluation of metastatic lesion in breast cancer. Peritoneal metastases are very lower incidence of breast cancer, but, in case of advanced metastatic breast cancer like this patient, peritoneal region could be metastatic sites. We present case with peritoneal metastasis including multiple another metastases.

\section{References}

1. Wong WY, Wong JS (2008) Uptake of Tc-99m methylene diphosphonate in peritoneal metastases from breast cancer. Clin Nucl Med 33(3): 218219.

\section{ISSN: 2574-1241}

DOI: 10.26717/BJSTR.2018.10.001899

Seok-Nam Yoon. Biomed J Sci \& Tech Res

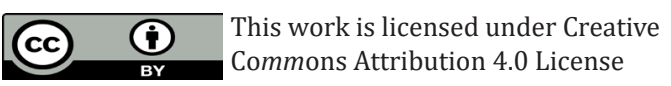

Submission Link: https://biomedres.us/submit-manuscript.php
2. Osaku T, Ogata H, Magoshi S, Kubota Y, Saito F, et al. (2015) Metastatic nonpalpable invasive lobular breast carcinoma presenting as rectal stenosis: a case report. J Med Case Rep 24(9): 88.

3. Saranovic D, Kovac JD, Knezevic S, Susnjar S, Stefanovic AD, et al. (2011) Invasive lobular breast cancer presenting an unusual metastatic pattern in the form of peritoneal and rectal metastases: a case report. J Breast Cancer 14(3): 247-250.

4. Sheen-Chen SM, Liu YW, Sun CK, Lin SE, Eng HL, et al. (2008) Abdominal carcinomatosis attributed to metastatic breast carcinoma. Dig Dis Sci 53(11): 3043-3045.

$\begin{array}{ll}\text { BIOMEDICAL } & \text { Assets of Publishing with us } \\ \text { RESEARCHES } & \text { - Global archiving of articles } \\ & \text { - Immediate, unrestricted online access } \\ & \text { - Rigorous Peer Review Process } \\ & \text { - Authors Retain Copyrights }\end{array}$

